\title{
Prevalence of suicidal behaviour following traumatic brain injury: Longitudinal follow-up data from the NIDRR Traumatic Brain Injury Model Systems
}

Lauren B. Fisher ${ }^{1,2}$, Paola Pedrelli 1,2, Grant L. Iverson ${ }^{3,4}$, Thomas F. Bergquist ${ }^{5}$, Charles H. Bombardier $^{6}$, Flora M. Hammond ${ }^{7,8}$, Tessa Hart ${ }^{9}$, Jessica M. Ketchum ${ }^{10}$, Joseph Giacino ${ }^{1,3,11}$, \& Ross Zafonte ${ }^{3,4,12,13}$

1. Department of Psychiatry, Massachusetts General Hospital , Boston, MA , USA.

2. Department of Psychiatry.

3. Department of Physical Medicine and Rehabilitation , Harvard Medical School , Boston , MA , USA.

4. Red Sox Foundation and Massachusetts General Hospital Home Base Program , Boston , MA , USA.

5. Departments of Psychology, Psychiatry, and Physical Medicine and Rehabilitation , Mayo Clinic College of Medicine at Rochester , Rochester , MN , USA.

6. Department of Rehabilitation Medicine, Division of Clinical and Neuropsychology , University of Washington, Seattle, WA, USA.

7. Department of Physical Medicine and Rehabilitation , Indiana University School of Medicine , Indianapolis , IN , USA.

8. Rehabilitation Hospital of Indiana, Indianapolis , IN , USA.

9. Moss Rehabilitation Research Institute , Elkins Park , PA, USA.

10. Department of Biostatistics \& Epidemiology , Georgia Regents University , Augusta , GA , USA.

11. Spaulding Rehabilitation Hospital, Boston, MA , USA.

12. Department of Physical Medicine and Rehabilitation, Massachusetts General Hospital , Boston, MA , USA.

13. Department of Physical Medicine and Rehabilitation , Brigham and Women's Hospital , Boston , MA , USA.

This is the author's manuscript of the article published in final edited form as:

Fisher, L. B., Pedrelli, P., Iverson, G. L., Bergquist, T. F., Bombardier, C. H., Hammond, F. M., ... Zafonte, R. (2016). Prevalence of suicidal behaviour following traumatic brain injury: Longitudinal follow-up data from the NIDRR Traumatic Brain Injury Model Systems. Brain Injury, 30(11), 13111318. https://doi.org/10.1080/02699052.2016.1195517 


\begin{abstract}
Objective: This study utilized the Traumatic Brain Injury Model Systems (TBIMS) National Database to examine the prevalence of depression and suicidal behavior in a large cohort of patients who sustained moderate to severe TBI.

Method: Participants presented to a TBIMS acute care hospital within 72 hours of injury, and received acute care and comprehensive rehabilitation in a TBIMS designated brain injury inpatient rehabilitation program. Depression and suicidal ideation were measured with the Patient Health Questionnaire (PHQ-9). Self-reported suicide attempts during the past year were recorded at each follow-up examination, at one, two, five, ten, fifteen, and twenty years postinjury.
\end{abstract}

Results: Throughout the twenty years of follow up, rates of depression ranged from 24.8 to 28.1\%, suicidal ideation ranged from 7.0 to $10.1 \%$, and suicide attempts (past year) ranged from 0.8 to $1.7 \%$. Participants who endorsed depression and/or suicidal behavior at year one demonstrated consistently elevated rates of depression and suicidal behavior five years after TBI. Conclusion: Compared to the general population, individuals with TBI are at greater risk for depression and suicidal behavior many years after TBI. The significant psychiatric symptoms evidenced by individuals with TBI highlight the need for routine screening and mental health treatment in this population.

Key words: traumatic brain injury, depression, suicide, suicidal ideation, suicide attempt, prevalence 
Introduction

Following traumatic brain injury (TBI), individuals face physical, cognitive, emotional, and behavioural consequences that often impair overall functioning, including independence, ability to work, and interpersonal relationships. Specifically, about $1.1 \%$ of the US population is living with long-term disability as a result of TBI [1], which often includes psychiatric disorders, most commonly depression [2]. Although the risk for developing Major Depressive Disorder (MDD) is significant in the first year after TBI [3], an elevated risk for depression persists across the lifespan. Reports of the prevalence of MDD after TBI have ranged from $6 \%$ to $77 \%$ using clinical rating scales [4] and from $17 \%$ to $61 \%$ when using structured diagnostic criteria [5]. The wide range of prevalence estimates is likely related in part to methodological differences in case definitions and measurement techniques. Different studies have used patient self-report rating scales (e.g. Patient Health Questionnaire-9 [6], Neurobehavioural Functioning Inventory [7]), relatives’ reports of symptoms, clinician-administered rating scales (e.g. Hamilton Depression Rating Scale [8]), and structured clinical interviews with established diagnostic criteria (e.g. Structured Clinical Interview for DSM-IV Diagnoses (SCID) [9]). Previously, 26\% of individuals enrolled in the Traumatic Brain Injury Model Systems National Database reported MDD one [10] and two years [11] after TBI. Seel and colleagues [12] demonstrated that 27\% of individuals with TBI met diagnostic criteria for MDD based on symptoms endorsed on the Neurobehavioural Functioning Inventory [7]. Five years after TBI, depression may be most prevalent for individuals aged 40 to 49 years relative to younger or older people [13]. Inconsistencies among reported prevalence of major depression following TBI is likely due to methodological differences not only in the variety of instruments used to assess symptoms but also variation in study design and sampling [3]. 
In addition to greater risk of depression, patients with TBI have elevated rates of suicidal behaviour compared to the general population [14]. Individuals who have sustained a TBI are 1.55 to 3 times more likely to complete suicide than individuals without TBI in the general population [15-17]. One study observed that over a five-year period suicide attempts were reported by $17.4 \%$ of individuals with history of mild-severe TBI, the majority without a preinjury history of suicidal behaviour [18]. Estimated rates of suicidal ideation following TBI have ranged from $22 \%$ to $28 \%$ [18-20]. Overall, severity of injury is not significantly related to the presence of suicidal behaviour (i.e. suicidal thoughts, suicide attempts) [18,20].

Little is known about the presence of both depression and suicidal behaviour in individuals who have sustained a TBI. In psychiatric populations, depressed individuals often experience suicidal ideation [21], which contributes to risk for suicide attempts and completed suicide [22]. In individuals with TBI, suicidal behaviour may be a symptom of depression or it may be related to pre-injury factors such as history of suicide attempt, bipolar disorder, less than high school education, and being on Medicaid [19]. In one study, about half of patients with TBI who reported suicidal ideation during the first year after injury also reported probable depression (PHQ-8 score $\geq 10$; PHQ-8 = PHQ-9 with suicide item excluded) at the time of their first assessment [19]. In another study, 26.1\% of participants who endorsed current suicidal ideation also met criteria for MDD [20].

Given the evidence of elevated rates of depression and suicidal behaviour in individuals with TBI, a more comprehensive examination of this topic is warranted. Most studies investigating suicidality in individuals with TBI have been limited to single sites, have collected data at only one time point, and have reported only on suicidal ideation rather than suicide attempts or suicide related deaths. Most studies were too small and time-limited to reliably 
examine rates of completed suicide. A large, multi-site study would add significantly to the existing knowledge base.

Thus, the current study used the TBI Model Systems National Database to examine rates of depression, suicidal ideation, suicide attempts, and completed suicide in a large cohort of patients who sustained moderate to severe TBI and underwent inpatient rehabilitation. We examined the prevalence of depression, suicidal ideation, suicide attempts, and completed suicide over 20 years following TBI that was severe enough to require inpatient rehabilitation. In addition, depression and suicidal behaviour were examined in subsets of individuals with greater risk for long term psychiatric problems (i.e. suicidal ideation present at year one, attempted suicide in the first year following TBI).

\section{Method}

\section{Participants}

Participants were enrolled in the National Institute on Disability and Rehabilitation Research (NIDRR) funded Traumatic Brain Injury Model Systems (TBIMS) National Database. Recruitment for TBIMS began in 1988 and contains longitudinal data from twenty current and previously-funded TBIMS centres throughout the US. All enrolled participants received inpatient rehabilitation after they sustained a TBI caused by an external mechanical force and experienced one of the following: (a) loss of consciousness exceeding 30 minutes (unless due to sedation or intoxication, (b) Glasgow Coma Scale (GCS) score less than 13 while in the emergency department (unless due to intubation, sedation, or intoxication), (c) PTA greater than 24 hours, or (d) positive neuroimaging consistent with acute trauma. Participants were 16 years of age or older at the time of the injury, presented to a TBIMS acute care hospital within 72 hours of the 
injury, and received acute care and comprehensive rehabilitation in a TBIMS designated brain injury inpatient rehabilitation programme.

The present study examined various subsets of this large, multi-site database that included the variables of interest. Sample sizes vary due to the fact that data collection methods changed various times since the beginning of the study, measures were added and deleted from the follow up battery, and many participants were lost to follow up over time. Analyses that examined depression and suicidal ideation utilized follow-up data collected from October 2007 through September 2013 ( $n=4,135$ participants completed year one follow up visit), when the PHQ-9 (described below) was administered at follow-up, and included all individuals who completed the PHQ-9 at one or more assessment points during this time period. Analyses that examined suicide attempts during the previous year utilized follow up data collected from January 1997 through September 2013 ( $n=$ 8,827 participants completed year one follow up visit). The number of completed suicides throughout the follow up period was examined among all available data from the beginning of recruitment in 1988 through September 2013 ( $n=12,046$ participants were enrolled in the study, number of follow up visits completed decreased each year due to attrition).

All database participants who completed at least one follow up visit from January 1997 through September 2013 were considered for analyses that included assessment of recent suicide attempts $(n=8,827)$. Of these participants, 57 were too cognitively or physically impaired to complete the first follow up assessment, 52 were not available at the first follow up, 11 refused to participate in the first follow up assessment, 35 were lost to follow up, and an additional 125 participants did not complete the recent suicide attempt question at year one for reasons that are 
unknown. The remaining 8,547 participants comprised the sample for analyses addressing suicide attempts.

Measures

Demographic variables included were age, sex, race, marital status, years of education, and occupational and employment status (pre and post injury). Pre-injury factors included presence or absence of pre-injury mental health treatment and psychiatric hospitalizations.

The Patient Health Questionnaire (PHQ-9) [6] is a 9-item, reliable and valid [23], selfadministered screening questionnaire used to assess depression. The items are based on the DSM-IV diagnostic criteria for major depression: (a) little pleasure or interest in doing things; (b) feeling down, depressed, or hopeless; (c) problems with sleep; (d) feeling tired or having little energy; (e) poor appetite or overeating; (f) feelings of worthlessness or guilt; (g) concentration problems; (h) psychomotor retardation or agitation; and (i) thoughts of suicide. Participants were asked to rate how often they were bothered by each symptom over the past two weeks: 0 (not at all), 1 (several days), 2 (more than half the days), or 3 (nearly every day). The PHQ-9 has been validated against a structured clinical interview for MDD in people with TBI [24]. Two scoring methods used to estimate the presence of MDD from the PHQ-9 are the “Depression-TBI” approach and the "Depression-Standard” approach. The Depression-TBI scoring method requires the endorsement of 5 or more depressive symptoms for at least several days over the last 2 weeks (score of $\geq 1$ on the PHQ-9), with at least one symptom being depressed mood or anhedonia. This approach resulted in a sensitivity of 0.93 and a specificity of 0.89 in diagnosing MDD among persons with TBI $[11,24]$. The Depression-Standard approach corresponds more closely to DSM-IV criteria and was included in the analyses given that it is more consistently used in psychiatry when screening for probable MDD. The Depression- 
Standard approach requires the presence of 5 or more depressive symptoms for more than half the days over the last 2 weeks (score of $\geq 2$ on the PHQ-9), with at least one symptom being depressed mood or anhedonia. This method resulted in lower sensitivity (0.60) but greater specificity (0.98) [24] compared to the "Depression-TBI” approach.

Suicidal Ideation. Suicidal ideation (SI) was assessed using the suicidal ideation item from the PHQ-9 which measures the frequency with which a participant has had "thoughts that you would be better off dead, or of hurting yourself' over the past two weeks. Scores of $\geq 1$ indicate the presence of suicidal ideation. Information about suicidal ideation was examined during the two weeks preceding each of the follow-up visits $(1,2,5,10,15$, and 20 years post injury).

Suicide Attempt. During the baseline interview, patients were asked to indicate if they ever attempted suicide in their lifetime (yes/no). At each follow-up visit, patients were asked if they attempted suicide at least once within the past year (yes/no). Thus, information was examined about the presence or absence of suicide attempt(s) in the year preceding each of the follow-up visits (1, 2, 5, 10, 15, and 20 years post injury).

Completed Suicide. If the patient was no longer living at the time of follow up assessment, TBIMS study coordinators obtained death certificates and coded the cause of death (including suicide) using ICD-9 codes. Deceased individuals assigned E-code classifications for suicide and self-inflicted injury, E950-E959, were considered to have completed suicide. Procedures

The TBIMS study was approved by institutional review boards at all TBIMS centres. Standardized data collection procedures were utilized by all TBIMS centres. Informed consent was obtained directly from patients or from a legal proxy. Once participants were enrolled in the 
TBIMS study, demographic data were obtained from medical records or participant interview. The Pre-injury History Questionnaire was used as a face-to-face interview, telephone interview, or mail-out survey to collect baseline data related to psychosocial history as soon as possible after the injury.

Follow up. Follow-up data were obtained by research staff using a standardized protocol via telephone or in person interview. If research staff were unable to reach participants, they were mailed standardized data collection forms with a self-addressed return envelope. Research staff attempted to obtain follow up data from every participant who was enrolled in the TBIMS study, unless the participant was reported as deceased or withdrew from the study. If a participant did not or could not respond to certain questions, a qualified proxy could respond for the patient. However, due to the focus on subjective experience of depression in the present study, follow up data provided by a proxy were not included in the current analyses. For the first year of follow up, interviews were conducted as close to the anniversary date of the injury as possible, with a window of \pm 2 months. For the second year of follow up, interviews were conducted as close to the anniversary date or the injury as possible, with a window of \pm 3 months. For follow up years 5 and thereafter, interviews were conducted as close to the anniversary date of the injury as possible, with a window of \pm 6 months.

\section{Statistical Analyses}

All statistical analyses were calculated using SPSS 22. Prevalence rates were calculated as the proportion of participants interviewed at a given assessment point, regardless of whether or not the variable had been reported at a previous assessment point. 


\section{Results}

The following sample description includes the 8,547 participants with data available on suicide attempts. Additional subsamples were even smaller subsets. At the time of injury, participants ranged in age from 16 to 99 years old (mean $\pm S D=39.9 \pm 18.8$ ). The majority of participants were men (72.9\%) and white (70.3\%). Most participants were single/never married (46.7\%) or married (33.5\%), while fewer participants were divorced (11.4\%), separated (3.7\%), or widowed (4.5\%). The majority of participants had obtained a high school education or less (60.5\%) and were competitively employed at the time of injury (63.1\%). Further, the most frequent causes of injury among this sample were motor vehicle accidents (40.8\%), falls (23.9\%), and motorcycle accidents (10.4\%). Although all patients sustained a moderate or severe TBI according to one of the four inclusion criteria listed above, $46.3 \%$ of participants obtained a Glasgow Coma Score (GCS) $\leq 8$ (indicative of severe TBI), 14.9\% obtained a GCS score in the range of 9 to 12 (indicative of moderate TBI), and $38.8 \%$ obtained a GCS score $\geq 13$ (in the range of mild TBI). Almost half (43.1\%) of participants reported problems with substance use prior to the injury. About $40 \%$ of participants reported abstaining from alcohol use, $19.3 \%$ were light drinkers, $24.6 \%$ were moderate drinkers, and 16.5\% were heavy drinkers. The majority of participants denied any prior history of mental health treatment (80.8\%), including prior psychiatric hospitalizations (95\%).

Rates of depression, suicidal ideation, and suicide attempts were examined during each completed follow up assessment up until twenty years post injury (see Table 1). Completed suicides were also identified at all follow up visits since the initiation of the study, between 1989 and September 2013. A total of 12,046 individuals were enrolled in the TBIMS study, and 21 completed suicides were identified over the course of 24 years. Specifically, there were 4 
completed suicides within the first year following TBI (0.03\%; $n=4 / 12,046), 6$ between year 1 and year 2 follow up (0.05\%; $n=6 / 12,046)$, 7 between year 2 and year 5 follow up $(0.06 \% ; n=$ 7/12,046), 2 between year 5 and year 10 follow up (0.02\%; $n=2 / 12,046), 2$ between year 10 and year 15 follow up (0.02\%; $n=2 / 12,046)$, and 0 between year 15 and year 20 follow up.

Percentages were calculated under the assumption that individuals who dropped out of the study and did not complete follow up assessments are still living. The cumulative rate of suicide over 20 years follow up was $0.17 \%(n=21 / 12,046)$.

A subset of participants with injuries between 1997 and September 2013 were asked to report history of suicide attempts prior to sustaining the brain injury for which they were enrolled in the national database. Out of 4,515 participants assessed, 4.3\% $(n=195)$ reported the presence of at least one prior suicide attempt. Out of the 195 individuals with prior histories of at least one suicide attempt, 7.6\% ( $n=10 / 131)$ reported a suicide attempt in the first year after the injury, $6.8 \%(n=7 / 103)$ reported a suicide attempt between years one and two after the injury, and 3.7\% ( $n=1 / 27$ ) reported a suicide attempt between years four and five after the injury. Further, rates of depression (using Depression-TBI scoring method) in individuals with pre-injury history of suicide attempt(s) were $46.4 \%(n=52 / 112)$ at year one follow up, 59.5\% $(n=50 / 84)$ at year two follow up, and $47.8 \%(n=11 / 23)$ at year five follow up, and rates of suicidal ideation were 26.8\% ( $n=30 / 112), 29.8 \%(n=25 / 84)$, and 13.0\% $(n=3 / 23)$, respectively.

Long-term prevalence of depression (using the Depression-TBI scoring method) and suicidal behaviour were also examined in individuals who endorsed depression and/or suicidal behaviour at the year one follow-up visit. Table 2 shows the prevalence of depression (87.6\%, 59\%, 58.4\%) and suicide attempts (8.1\%, 3.6\%, 8.8\%) at follow up years one, two, and five, respectively, among individuals who endorsed the presence of suicidal ideation at year one 
follow up ( $n=322$ ). Of those individuals who reported suicidal ideation at the year one follow up and completed another follow up visit(s), 35.5\% (83/234) continued to report suicidal ideation at year 2 and 27.3\% (21/77)) at year 5. Table 3 shows the prevalence of depression (66\%, 59.2\%, 42.9\%) and suicidal ideation (55.3\%, 28.6\%, 20.9\%) at follow up years one, two, and five, respectively, among individuals who endorsed at least one suicide attempt in the first year after TBI $(n=114)$. Of those individuals who reported at least one suicide attempt in the first year after TBI and completed another follow up visit(s), 17.6\% (16/91) reported another suicide attempt in the second year after TBI and 7.7\% (4/52) reported another suicide attempt between years four and five following TBI. Finally, Table 4 shows the prevalence of suicidal ideation (31.2\%, 21.1\%, 15.1\%) and suicide attempts (3.5\%, 2.4\%, 4.3\%) at follow up years one, two, and five, respectively, among individuals who endorsed the likely presence of MDD at one year post-TBI $(n=895)$. Of those individuals who reported MDD at the year one follow up and completed another follow up visit(s), 58.8\% (367/624) continued to report MDD at year 2 and $52.9 \%(101 / 191)$ at year 5 .

\section{Discussion}

Compared to the general population, rates of MDD, suicidal ideation, and suicide attempts are higher following TBI and remain consistently elevated for up to twenty years following the injury. In the current sample, throughout the twenty years of follow up, rates of screening positively for probable MDD ranged from 24.8 to $28.1 \%$, defined according to the Depression-TBI scoring method recommended for individuals with TBI [24], and from 10.2\% to 14.8\% using the Depression-Standard scoring method that is used in some studies in psychiatry and the general population. Depending on the PHQ-9 scoring approach, these rates are considerably higher than the 12-month prevalence estimates of MDD observed in the general 
population based on structured diagnostic interviews (6.6\% [25]; 5.3\% [26]). Using the Depression-TBI scoring method, rates of MDD in the present study were very similar to the rates previously reported by Hart et al. [10-11] using the same scoring method, one and two years after TBI (26\%). In addition, current findings were similar to point prevalence rates reported throughout the first year after injury (21-31\% [3]). Rates of MDD (using the PHQ-9) after TBI in the present study were similar, if not higher, than in patients with spinal cord injury one to five years following injury (18-21\% [27]).

Throughout the twenty years assessed, rates of suicidal ideation (in the past two weeks) were 2 to 3 times higher in the current study (7.0-10.1\%) than 12-month prevalence in the general population (2.8\% [28]); however, they were somewhat lower than rates of suicidal ideation in individuals with spinal cord injury (15.4\% [29]). Similarly, rates of suicide attempts in the past year reported at each follow-up assessment were at least two times higher in the present sample (1.1-1.7\%) than in the general population (0.6\% [25]) during years $1-15$, but more similar at 20 years following injury (0.8\%). Current findings are consistent with previous reports of elevated rates of suicidal behaviour in adults living with TBI in the community [20], outpatients receiving rehabilitation services [18], and patients who were prospectively followed after hospitalization for TBI [19]. It is important to note that compared to other studies in TBI, our study revealed lower rates of suicidal ideation, which may best be explained by the varying time frames in which suicidal ideation was assessed. In our follow up visits, participants were only asked to report the presence of suicidal ideation at one time point within the past two weeks, whereas analyses for other studies reported the presence of suicidal ideation over longer periods of time, such as within the past two weeks at various time points across one year [19] and five years [20]. 
Recent data from the Centers for Disease Control and Prevention highlight an increasing and alarming trend in suicide deaths. The age adjusted rate for suicide in the general population in 2014 was 13.0 per 100,000 population, a $24 \%$ increase from the rate in 1999 [30]. The rate of completed suicide in our study was higher, ranging from $0.02-0.06 \%$ across the twenty years of follow up. Other studies in TBI have shown even higher rates of suicide in the first year after injury (e.g. 0.6\% [16]) and up to fifteen years following TBI (e.g. 0.59-0.84\% [31]).

Our examination of suicidal behaviour and depression during the first five years following TBI revealed a number of interesting findings. The majority of individuals with suicidal ideation at year one were likely to meet criteria for MDD (87.6\%) and almost one out of ten (8.1\%) of them reported a suicide attempt in the first year after TBI. Relative to the general population, 38.9-41.9\% of individuals who report suicidal ideation in the last 12 months meet criteria for MDD in the past year [28], which is notably less than in the current sample. Further, 12-month suicide attempt rates among individuals with suicidal ideation are 7.3-9.7\% [28], which is comparable to the proportion of current ideators in the present sample who report a suicide attempt in the first year after injury. When comparing our findings with those of Kessler et al., [28], it is important to note the differences in time frame assessed, as well as definitions of suicidal ideation used in each study. Although the rates of depression, suicidal ideation, and suicide attempts in the current study appear to drop off notably at year two, they remain somewhat stable and elevated at year five. This finding suggests a longitudinal pattern of chronic problems in individuals who displayed suicidal ideation one year after TBI, which argues for the need to intervene when individuals display early signs of suicidal ideation. Further, the number of individuals with suicidal ideation who also meet criteria for MDD is higher than previous findings [19, 20], suggesting that depression plays a significant role in the presence of suicidal 
thoughts one year after TBI. By treating MDD in individuals with TBI, it may be possible to reduce suicidal ideation. Furthermore, findings suggest that suicidal ideation in this population may be largely conceptualized as a symptom of depression rather than a distinct neuropsychiatric sequela of TBI.

Similar to the findings discussed above, more than half of individuals who reported at least one suicide attempt in the first year following TBI were likely to meet criteria for MDD (66\%) and report suicidal ideation (55.3\%) at year one follow-up. To compare, 38.9\% of individuals who report at least one suicide attempt in the last 12 months meet criteria for MDD in the general population [28], which is notably lower than the proportion of recent attempters who met criteria for probable MDD in the present study. Individuals with suicide attempt(s) in the first year after TBI also had consistently elevated rates of MDD and suicidal ideation by years two and five. Furthermore, individuals who attempted suicide in the first year following TBI had alarmingly high rates of subsequent suicide attempt(s) after two years (17.6\%). Although the prevalence of MDD among individuals who attempt suicide in the first year following TBI is high, it is comparatively lower than the rate of MDD among individuals with suicidal ideation at year one, suggesting that other factors (i.e. impulsivity, low frustration tolerance) may play an important role in predicting who attempts suicide in the first year following TBI. However, it may be that suicide attempters were more likely to receive psychiatric treatment in the context of post-attempt hospitalization, whereas suicide ideators were less likely to receive psychiatric treatment. Finally, more than half of individuals with MDD at year one displayed evidence of MDD two and five years following TBI with somewhat more elevated rates of suicidal ideation and suicide attempts compared to the entire sample. Similarly, Hart et al. [11] previously found that three-fourths of those with MDD at year one 
experienced clinically significant depressive symptoms at year 2. Taken together, individuals with MDD and/or suicidal behaviour one year following TBI appear to be at greater risk for chronic psychiatric problems up to five years after TBI.

A small percentage of individuals in the present study reported a history of suicide attempt(s) prior to sustaining a TBI (4.3\%). Compared to the entire sample, the individuals with pre-injury suicide attempt(s) went on to demonstrate higher rates of suicide attempts, suicidal ideation, and depression in the five years following TBI. A similar trend was noted in this subsample of at-risk individuals wherein risk for subsequent suicidal behaviour was similarly heightened in the first two years after TBI and then declined notably five years after the injury.

Previous research has demonstrated that individuals who have sustained a TBI are at significant risk for depression in the first year after the injury [3], and our study suggests that this is also true for suicidal behaviour, with the period of significant risk possibly extending into the second year after the injury. The current research expands upon recent findings on suicidal ideation after TBI by Mackelprang et al. [19] by utilizing a large, multi-site, cross-national sample. In addition, the current findings include longitudinal assessment of suicide attempts, in addition to suicidal ideation.

The present findings should be considered in light of potential limitations. Suicidal ideation was assessed using a single item from the PHQ-9 which does not distinguish between active and passive ideation, suicide intent, and suicide plans. Although the suicide ideation item of the PHQ-9 appears to be an adequate screener in detecting suicidal ideation, sensitivity (0.69) is not high [32] so there are likely some false negatives in this study. Assessment of recent suicide attempts relied on a single question worded in such a way that it only captured suicide attempts that occurred in the past year. After the year 2 follow-up, assessments were not 
completed yearly, meaning that data on some suicide attempts may have been missed. In addition, some individuals may have experienced motor impairment that prevented them from otherwise acting on suicidal ideation. Similar to past studies on completed suicides, some cases were likely missed. Suicides are underreported and sometimes misclassified by medical examiners due to incomplete data collection in the initial investigation of the death, ambiguous circumstances surrounding the death, such as single car accidents ruled as accidental when they were suicides [33], or the deceased having taken steps to disguise the suicide [34]. Further, psychiatric treatment was not tracked, and thus controlling for treatment was not possible. In addition, pre-injury depression was not tracked, so we were unable to contrast suicidal behaviour in patients with and without pre-injury depression. The inconsistent entry and removal of study variables throughout the course of the twenty year study limits the observations that can be made between various subgroups and led to subsamples of unequal sizes. Finally, the current study was strictly descriptive, and thus we draw no conclusions as to causality or prediction.

The current findings expand upon our previous knowledge of suicidal behaviour many years following TBI. Individuals with TBI are at notably greater risk for depression and suicidal behaviour than the general population, and individuals who sustain a TBI and endorse a history of pre-injury suicide attempt(s) as well as depression and suicidal behaviour in the first year after injury continue to be at even greater risk for continued psychiatric problems throughout the first five years following TBI. Future research will examine predictors of suicidal behaviour following TBI. Given our current findings, it is likely that different factors may predict which individuals experience suicidal ideation and which individuals go on to attempt suicide. Regardless, the significant psychiatric symptoms evidenced by individuals with TBI highlight an important need for mental health treatment in this population. Reasons for increased rates of 
depression and suicidal behaviour as many as two years after the injury are unclear, though difficulties adjusting to life following TBI, returning to work, and engaging in one's environment may be at play. In addition, pre-injury psychiatric problems (i.e. pre-injury suicide attempts) also seem to play a role in increased rates of depression and suicidal behaviour many years after TBI. Importantly, pre-injury psychiatric conditions can easily be identified, providing early opportunities for monitoring and intervention. Given that individuals in the current study received intensive, multidisciplinary treatment in the months following the injury, it is possible that the psychological effects of living with a TBI do not set in until longer after the acute medical crisis is passed. Routine screening and referrals for treatment when needed should be a staple in follow up appointments in brain injury outpatient clinics. 


\section{References}

[1] Zaloshnja E, Miller T, Janglois JA, Selassie AW. Prevalence of long-term disability from traumatic brain injury in the civilian population of the United States. J Head Trauma Rehabil. 2008;23:394-400.

[2] Koponen S, Taiminen T, Portin R, Himanen L, Isoniemi H, Heinonen H, Hinkka S, Tenovuo O. Axis I and II disorders after traumatic brain injury: A 30-year follow-up study. Am J Psychiatry. 2002;159: 1315-1321.

[3] Bombardier CH, Fann JR, Temkin NR, Esselman PC, Barber J, Dikmen SS. Rates of major depressive disorder and clinical outcomes following traumatic brain injury. JAMA. 2010;303: 1938-1945.

[4] Jorge RE, Starkstein SE. Pathophysiologic aspects of major depression following traumatic brain injury. J Head Trauma Rehabil. 2005;20:475-487.

[5] Rapoport MJ. Depression following traumatic brain injury. CNS Drugs. 2012;26:111-121.

[6] Kroenke K, Spitzer R, Williams J. The PHQ-9: Validity of a brief depression severity measure. J Gen Intern Med. 2001;16:606-613.

[7] Kreutzer JS, Marwitz JH, Seel R, Serio CD. Validation of a neurobehavioural functioning inventory for adults with traumatic brain injury. Arch Phys Med Rehabil. 1996;77:116-124.

[8] Hamilton MA. A rating scale for depression. J Neurol Neurosurg Psychiatry. 1960;23:56-62.

[9] First MB, Spitzer RL, Gibbon M, Williams JBW. 1995. Structured Clinical Interview for DSM-IV-TR Axis I Disorders, Research Version, Patient Edition. (SCID-I/P) New York: Biometrics Research, New York State Psychiatric Institute, November 2002.

This is the author's manuscript of the article published in final edited form as:

Fisher, L. B., Pedrelli, P., Iverson, G. L., Bergquist, T. F., Bombardier, C. H., Hammond, F. M., ... Zafonte, R. (2016). Prevalence of suicidal behaviour following traumatic brain injury: Longitudinal follow-up data from the NIDRR Traumatic Brain Injury Model Systems. Brain Injury, 30(11), 13111318. https://doi.org/10.1080/02699052.2016.1195517 
[10] Hart T, Brenner L, Clark AN, Bogner JA, Novack TA, Chervoneva I, Nakase-Richardson R, Arango-Lasprilla JC. Major and minor depression after traumatic brain injury. Arch Phys Med Rehabil. 2011;92:1211-9.

[11] Hart T, Hoffman JM, Pretz C, Kennedy R, Clark AN, Brenner LA. A longitudinal study of major and minor depression following traumatic brain injury. Arch Phys Med Rehabil. 2012;93:1343-1349.

[12] Seel RT, Kreutzer JS, Rosenthal M, Hammond FM, Corrigan JD, Black K. Depression after traumatic brain injury: A National Institute on Diability and Rehabilitation Research Model Systems multicenter investigation. Arch Phys Med Rehabil. 2003;84:177-184.

[13] Corrigan JD, Cuthbert JP, Harrison-Felix C, Whiteneck GG, Bell JM, Miller AC, Coronado VG, Pretz CR. US population estimates of health and social outcomes 5 years after rehabilitation for traumatic brain injury. J Head Trauma Rehabil. 2014;29:E1-E9.

[14] Simpson G, Tate R. Suicidality in people surviving a traumatic brain injury: Prevalence, risk factors, and implications for clinical management. Brain Inj. 2007;21:1335-1351.

[15] Brenner LA, Ignacio RV, Blow FC. Suicide and traumatic brain injury among individuals seeking Veterans Health Administration services. J Head Trauma Rehabil. 2011;26(4):257264.

[16] Harrison-Felix CL, Whiteneck GG, Jha A, DeVivo MJ, Hammond FM, Hart DM. Mortality over four decades after traumatic brain injury rehabilitation: A retrospective cohort study. Arch Phys Med Rehabil. 2009;90:1506-1513.

[17] Ventura T, Harrison-Felix C, Carlson N, Diguiseppi C, Gabella B, Brown A, Devivo M, Whiteneck G. Mortality after discharge from acute care hospitalization with traumatic brain injury: A population-based study. Arch Phys Med Rehabil. 2010;91:21-9. 
[18] Simpson G, Tate R. Suicidality after traumatic brain injury: Demographic, injury and clinical correlates. Psychol Med. 2002;32:687-697.

[19] Mackelprang JL, Bombardier CH, Fann JR, Temkin NR, Barber JK, Dikmen SS. Rates and predictors of suicidal ideation during the first year after traumatic brain injury. Am J Public Health. 2014;104:e100-e107.

[20] Tsaousides T, Cantor J, Gordon W. Suicidal ideation following traumatic brain injury: Prevalence rates and correlates among adults living in the community. J Head Trauma Rehabil. 2011;26:265-275.

[21] Möller HJ. Suicide, suicidality and suicide prevention in affective disorders. Acta Psychiatr Scand. 2003;108:73-80.

[22] Nock MK, Borges G, Bromet EJ, Cha CB, Kessler RC, Lee S. Suicide and suicidal behavior. Epidemiol Rev. 2008;30:133-154.

[23] Kroenke K, Spitzer RL, Williams JB, Lowe B. The patient health questionnaire somatic, anxiety, and depressive symptom scales: A systematic review. Gen Hosp Psychiatry. 2010; 32:345-359.

[24] Fann J, Bombardier C, Dikmen S, Esselman P, Warms CA, Pelzer E, Rau H, Temkin N. Validity of the Patient Health Questionnaire-9 in assessing depression following traumatic brain injury. J Head Trauma Rehabil. 2005;20:501-511.

[25] Kessler RC, Berglund P, Demler O, Jin R, Koretz D, Merikangas KR, Rush AJ, Walters EE, Wang PS. The epidemiology of major depressive disorder. Results from the National Comorbidity Survey Replication (NCS-R). JAMA. 2003;289:3095-3105.

[26] Hasan D, Goodwin RD, Stinson FS, Grant BF. Epidemiology of major depressive disorder. Results from the National Epidemiologic Survey on Alcoholism and Related Conditions. 
Arch Gen Psychiatry. 2005;62:1097-1106.

[27] Hoffman JM, Bombardier CH, Graves DE, Kalpakjian CZ, Krause JS. A longitudinal study of depression from 1 to 5 years after spinal cord injury. Arch Phys Med Rehabil. 2011;92:411-418.

[28] Kessler RC, Berglund P, Borges G, Nock M, Wang PS. Trends in suicide ideation, plans, gestures, and attempts in the United States, 1990-1992 to 2001-2003. JAMA. 2005;293(20):2487-2495.

[29] Bombardier CH, Richards JS, Krause JS, Tulsky D, Tate DG. Symptoms of major depression in people with spinal cord injury: implications for screening. Arch Phys Med Rehabil. Nov 2004;85(11):1749-1756.

[30] Curtin SC, Warner M, Hedegaard H. Increase in suicide in the United States, 1999-2014. NCHS data brief, no 241. Hyattsville, MD: National Center for Health Statistics. 2016. [31] Teasdale TW, Engberg AW. Suicide after traumatic brain injury: a population study. J Neurol Neurosurg Psychiatry. 2001;71:436-440.

[32] Uebelacker LA, German NM, Gaudiano BA, Miller IW. Patient Health Questionnaire depression scale as a suicide screening instrument in depressed primary care patients: A cross-sectional study. Prim Care Companion CNS Disord. 2011;13(1): PCC.10m01027.

[33] Linsley K, Schapira K, Kelly T. Open verdict v. suicide: Importance to research. Br J Psychiatry. 2001;178:465-468.

[34] Phillips DP, Ruth TE. (1993). Adequacy of official suicide statistics for scientific research and public policy. Suicide Life Threat Behav. 1993;23:307-319. 
Table 1

Rates of Depression, Suicidal Ideation, and Suicide Attempts Following Traumatic Brain Injury

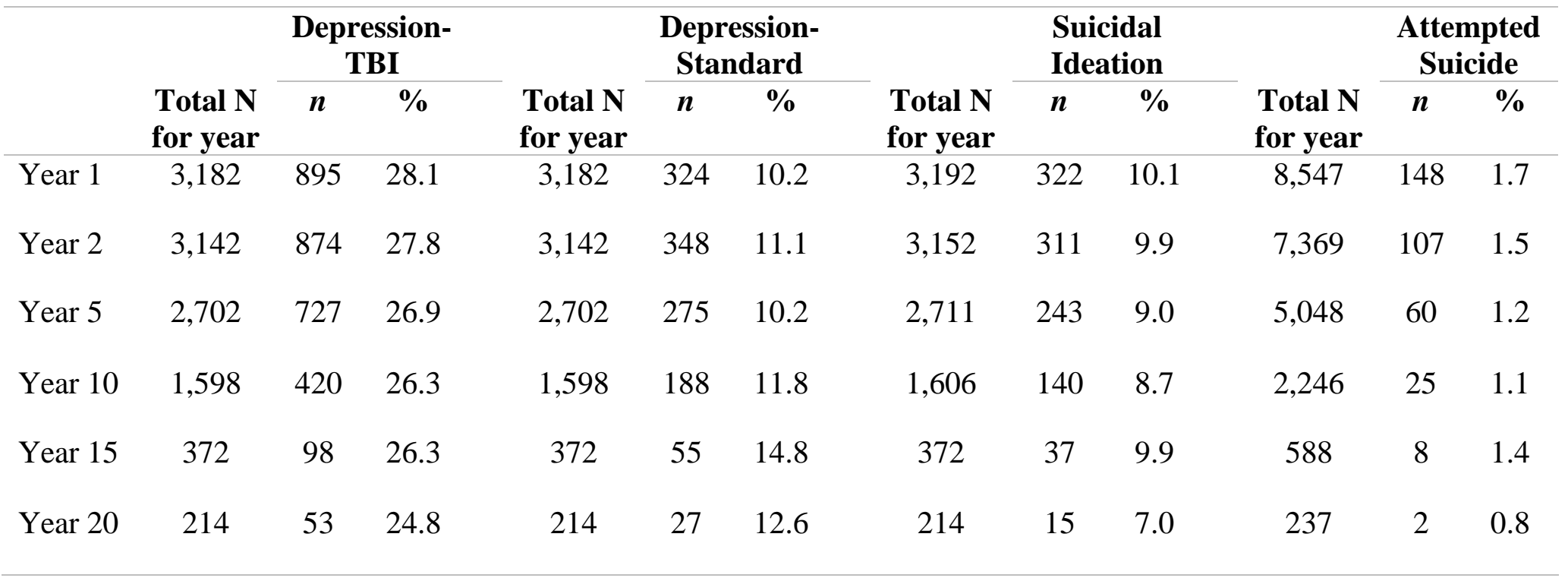

Note. The Total $\mathrm{N}$ for each year represents the total sample size per year for each variable.

Depression-TBI indicates the presence of 5 or more depressive symptoms for at least several days over the last 2 weeks (score of $\geq 1$ on the PHQ-9), with at least one symptom being depressed mood or anhedonia.

Depression-Standard indicates the presence of 5 or more depressive symptoms for more than half the days over the last 2 weeks (score of $\geq 2$ on the PHQ-9), with at least one symptom being depressed mood or anhedonia.

Suicidal Ideation indicates the presence of "thoughts that you would be better off dead, or of hurting yourself” for at least several days over the past 2 weeks (score of $\geq 1$ on the PHQ-9 suicide item). 
Depression-TBI, Depression-Standard, and Suicidal Ideation are calculated based on data collected after October 2007 (when PHQ-9 was added to study) through September 2013. Attempted Suicide rates (past year) are calculated based on data collected from 1997 through September 2013. 
Table 2

Long-Term Rates of Depression and Suicide Attempts in Participants with Suicidal Ideation One Year Following TBI $(n=322)$

\begin{tabular}{cccc}
\hline & Year 1 & Year 2 & Year 5 \\
& \multicolumn{3}{c}{ Suicide Attempts } \\
Suicide Attempt (SA) & $8.1 \%$ & $3.6 \%$ & $8.8 \%$ \\
& $(26 / 320)$ & $(9 / 252)$ & $(7 / 80)$ \\
\hline & & Major Depression \\
Major Depression (MDD) & $(287.6 \%$ & $59.0 \%$ & $58.4 \%$ \\
& & $(138 / 234)$ & $(45 / 77)$ \\
\hline
\end{tabular}


Table 3

Long-Term Rates of Depression and Suicidal Ideation in Participants with Recent Suicide Attempts One Year Following TBI $(n=114)$

\begin{tabular}{cccc}
\hline & Year 1 & Year 2 & Year 5 \\
& & Suicidal Ideation \\
& & & \\
Suicidal Ideation (SI) & $55.3 \%$ & $29.2 \%$ & $20.9 \%$ \\
& $(26 / 47)$ & $(14 / 48)$ & $(9 / 43)$ \\
\hline & & Major Depression \\
Major Depression (MDD) & $66.0 \%$ & $60.4 \%$ & $42.9 \%$ \\
& $(31 / 47)$ & $(29 / 48)$ & $(18 / 42)$ \\
\hline
\end{tabular}

Note. This analysis included only individuals who reported a suicide attempt at year 1 and who completed at least one follow up assessment after October 2007. 
Table 4

Long-Term Rates of Suicide Attempts and Suicidal Ideation in Participants with Major

Depression One Year Following TBI $(n=895)$

\begin{tabular}{lccc}
\hline & Year 1 & Year 2 & Year 5 \\
\hline & \multicolumn{3}{c}{ Suicidal Ideation } \\
Suicidal Ideation (SI) & $31.2 \%$ & $21.1 \%$ & $15.1 \%$ \\
& $(279 / 895)$ & $(131 / 622)$ & $29 / 192)$ \\
\hline & & Suicide Attempt \\
Suicide Attempt (SA) & $3.5 \%$ & $2.4 \%$ & $4.3 \%$ \\
& $(31 / 889)$ & $(16 / 675)$ & $9 / 207)$ \\
\hline
\end{tabular}

